\title{
Role of aluminum adjuvant in producing an allergic rhinitis animal model
}

\author{
L. Xi ${ }^{1,2}$, E. Fan ${ }^{2}$, Y. Zhao ${ }^{1,2}$, Y. Li ${ }^{2}$, Y. Zhang ${ }^{1,2}$ and L. Zhang ${ }^{1,2}$ \\ ${ }^{1}$ Department of Otolaryngology, Head and Neck Surgery, \\ Beijing Tongren Hospital, Capital Medical University, Beijing, China \\ ${ }^{2}$ Key Laboratory of Otolaryngology, \\ Head and Neck Surgery, Ministry of Education of China \\ Beijing Institute of Otolaryngology, Beijing, China \\ Corresponding author: L. Zhang \\ E-mail: luozhangcn@163.com
}

Genet. Mol. Res. 13 (3): 5173-5181 (2014)

Received May 16, 2013

Accepted September 20, 2013

Published July 7, 2014

DOI http://dx.doi.org/10.4238/2014.July.7.10

\begin{abstract}
This study evaluated different dosage forms of aluminum adjuvant in generating allergic rhinitis animal models. Forty female BALB/c mice were assigned to four groups, including three dosage forms of aluminum adjuvant [powder, gel, and hydrosolvent of aluminum hydroxide, $\left.\mathrm{Al}(\mathrm{OH})_{3}\right]$ mixed with ovalbumin to simulate the symptoms of allergic rhinitis and one control group. Although the aluminum adjuvants were in different dosage forms, the content was 5 mg after conversion in all groups. The fourth group was given normal saline instead as a control. Mice of the powder group displayed typical symptoms of allergic rhinitis. We also found discrete eosinophils in the nasal mucosa of mice from the hydrosolvent group; however, no eosinophils were found in the gel group. These two groups both displayed cytotoxic symptoms and foreign body granuloma. Aluminum adjuvant used in producing animal models can induce foreign body granuloma and other untoward reactions, which are associated with the
\end{abstract}


dosage level and form.

Key words: Aluminum adjuvant; Animal model; Allergic rhinitis; Foreign body granuloma; Colloid chemistry

\section{INTRODUCTION}

Immunoadjuvants are commonly used to reinforce the immunogenicity of an antigen in inducing an allergic rhinitis (AR) animal model. The ideal immunoadjuvant must promote the antigen to generate classical symptoms and pathological changes without inducing untoward reactions. Because of their good adsorbability, aluminum adjuvants can be used to induce humoral immunity reactions and stimulate T-helper2 (Th2) cells to generate a high level of antibodies, and are thus the first choice as immunoadjuvants. However, different dosage forms and methods applying aluminum adjuvants have yielded different results in different experiments. Inevitably, unexpected damage on organs will often disturb such studies. This study focused on the issue of using an aluminum adjuvant to establish an AR animal model and the comparison of three different dosage forms of aluminum adjuvant.

\section{MATERIAL AND METHODS}

\section{Animals}

The BALB/c mice were provided by the animal center of Capital Medicine University, and were maintained in a specific pathogen free environment. All forty mice were females in good health, 6 to 8 weeks of age, and ranged in weight from 17 to $24 \mathrm{~g}$. This study was carried out in strict accordance with the recommendations in the Guide for the Care and Use of Laboratory Animals of the National Institutes of Health. The animal use protocol was reviewed and approved by the Institutional Animal Care and Use Committee (IACUC) of Capital Medicine University.

\section{Grouping and establishment of AR animal model}

We used $50 \mu \mathrm{g}$ ovalbumin (OVA) blended with $5 \mathrm{mg}$ different dosage forms of aluminum hydroxide $\left[\mathrm{Al}(\mathrm{OH})_{3}\right]$ as a sensitogen to produce the animal models of AR (de Serres, 1988). The forty mice were randomly assigned to four groups by the drawing lots method. The first group was treated with $50 \mu \mathrm{g}$ OVA and $5 \mathrm{mg} \mathrm{Al}(\mathrm{OH})_{3}$ powder. The second group was treated with $50 \mu \mathrm{g}$ OVA and $0.385 \mathrm{~mL}$ aluminum hydroxide gel. The third group was treated with $50 \mu \mathrm{g}$ OVA and $0.125 \mathrm{~mL}$ injected aluminum. The fourth group was treated with $0.9 \%$ sodium chloride instead, and acted as the control group. The animal model was produced in two stages: sensitization and provocation. In the first stage, mice in the case groups (groups 1 to 3) were injected with $50 \mu \mathrm{g}$ OVA and $5 \mathrm{mg} / \mathrm{mL} \mathrm{Al}(\mathrm{OH})_{3}$ through an enterocoelic injection every two days for a total of seven times while mice in the control group were treated with $0.9 \%$ sodium chloride instead. The provocation stage started at the end of the sensitization stage. Mice in the case groups were nasally administrated $20 \mu \mathrm{L}$ OVA in $10 \mu \mathrm{L} 0.9 \%$ sodium chloride in each nasal cavity and the control group was administrated $0.9 \%$ sodium chloride 
only. At the same time, we observed and recorded behaviors and symptoms. Twenty-four hours after the last provocation treatment, mice were scarified by cervical dislocation and the necessary tests were conducted.

\section{Electron microscopy observations of pathology and transmission}

The nasal cavities of mice were removed and were fixed in neutral methanol for $24 \mathrm{~h}$. After using 10\% EDTA for decalcification for 3 weeks, $4 \mu \mathrm{m}$ nasal cavity coronal slices was embedded in paraffin and stained with hematoxylin and eosin (HE) for microscope observations. The abdominal cavities of all mice were observed for histopathological assessments.

The granulation tissue on the surfaces of the liver and spleen was observed through transmission electron microscopy in the second group of mice. Several $1 \mathrm{~mm}^{3}$ sections living tissue were immediately fixed in $2.5 \%$ glutaraldehyde, rinsed with phosphate-buffered saline (PBS), fixed in osmic acid, dehydrated in acetone step by step, embedded in EP-812, and then were made into ultrathin sections, stained with lead acetate and sodium citrate, and then features were examined under a JME-1010 transmission electron microscope. At the same time, the chemical constituent of granuloma was examined by JEM-2100F industry transmission electron microscopy and energy spectrum analysis.

\section{RESULTS}

\section{Physical examination and behavioral observations}

The appearance of mice in the first group was normal, and after basic sensitization and challenging, the most frequently observed symptoms included nose scratching, rhinorrhoea, sneezing, and swelling of the mucosa around the nasal cavity. The mice in the second group showed enlargement of the abdominal region, slowed movement, dull fur, and these mice became thin and emaciated. Administration of celiocentesis resulted in hemorrhagic ascites (Figure 1A). However, this group did not show classic symptoms after sensitization and challenging. Interestingly, 5 of the 10 mice of the third group showed ascites (Figure 1B) and their general conditions were better than those of the second group. In the sensitization stage, nose scratching and rhinorrhea were evident. The fourth group of mice (controls) showed no changes in behavior.

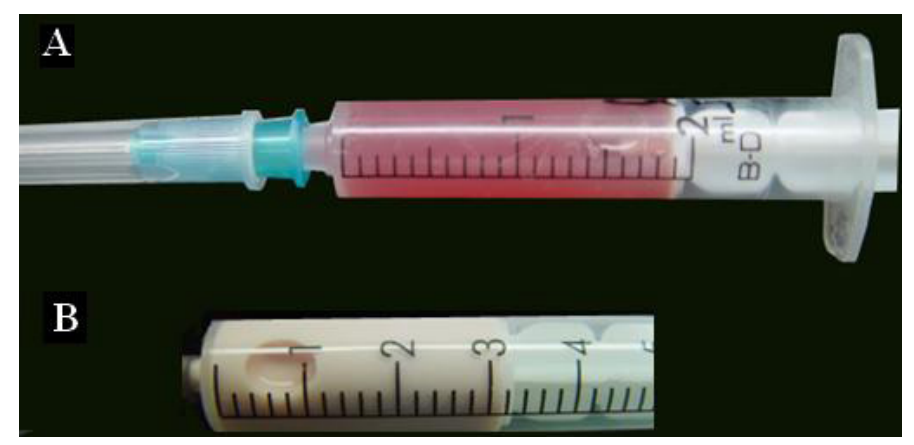

Figure 1. A. Second group $\left[\mathrm{Al}(\mathrm{OH})_{3}\right.$ gel $]=$ Bloody ascites from the abdominocentesis. B. Third group $\left[\mathrm{Al}(\mathrm{OH})_{3}\right.$ hydrosolvent $]=$ the chylia ascites. 


\section{Observation of nasal mucosa under HE staining}

In the first group $\left[\mathrm{Al}(\mathrm{OH})_{3}\right.$ powder], there were abundant eosinophils aggregating at the lateral nasal walls and in the mucosa of the inferior turbinate (Figure 2A). Mucosa and goblet cells showed obvious proliferation and active secretion. Staining with Alcian blueperiodic acid Schiff (AB-PAS) revealed a large quantity of acid mucopolysaccharides in goblet cells. Lymphocyte and neutrophil infiltration and the quantity of plasmocytes increased in the lamina propria. In the second group $\left[\mathrm{Al}(\mathrm{OH})_{3}\right.$ gel], there were no eosinophils found in the nasal mucosa of the mice. Cilia were arranged in a disorderly manner and there were some coloboma in the nasal mucosa. In the third group $\left[\mathrm{Al}(\mathrm{OH})_{3}\right.$ hydrosolvent], eosinophils were occasionally found in the nasal mucosa. In the fourth group (control), the nasal mucosa was intact and the ciliated epithelium lined up. There were discrete goblet cells and a small amount of eosinophils. The basement membrane was intact (Figure 2B).

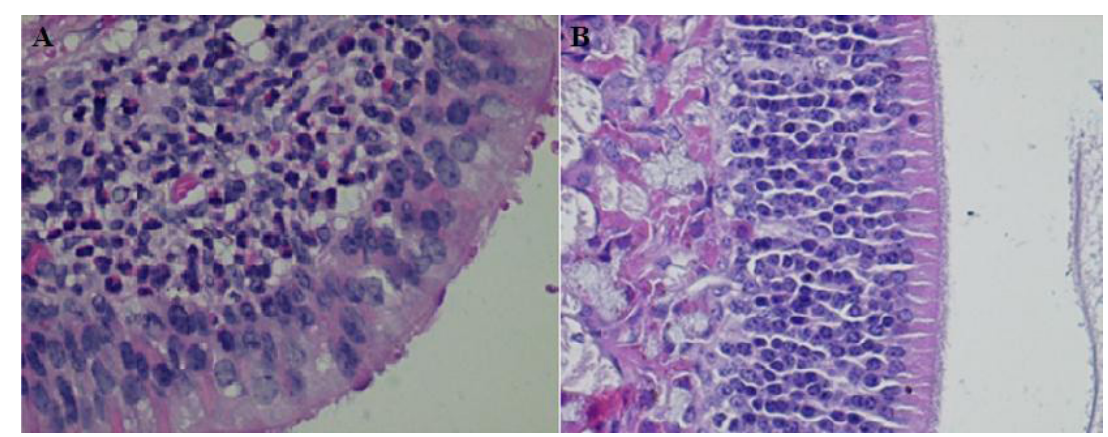

Figure 2. A. First group $\left[\mathrm{Al}(\mathrm{OH})_{3}\right.$ powder $]=$ there were eosnophiles in the nasal inferior Turbinate (40X). B. Control group $=$ the normal nasal cavity and nasal septum (40X).

\section{Coelom pathological examination}

In the first group, the mice showed normal activity and flesh fur. There were no abnormalities found in the examination of the viscera. In the second group, the structure of the hepatic lobule was clear, whereas foreign body granuloma emerged inside the tunication of the liver (Figure 3A). A granular gray substance was found in the granuloma cells and it showed a strong refraction (Figure 3B). Moreover, halos could also be seen around some of this substance. Similarly, the splenci cortex nodule atrophied, granuloma was found in the tunication of the spleen, and there were many neutrophils in the areas combined of the granuloma and spleen. Discrete polymorphonuclear macrophagocytes emerged in the splenic sinus. The structure of all levels of bronchia, alveola, and alveolar ducts were clear, but hyperemia of the alveolar septum capillary was obvious. A small amount of neutrophils and eosinophils were found in the bronchial mucosa. The structures of the glomerulus and renal tubule were clear. Early stage granuloma emerged in the retroperitoneum of the kidney. Except for some granuloma emerging in the lobule, the structure of the thymus was normal. In the third group, the focal granuloma was the same as that of the second group, and emerged in the liver, spleen, and kidney, and some lymphocytes and eosinophils were also found in the mesenchyme. In the fourth group, the pathological examinations of the liver, spleen, and kidney were all normal. 


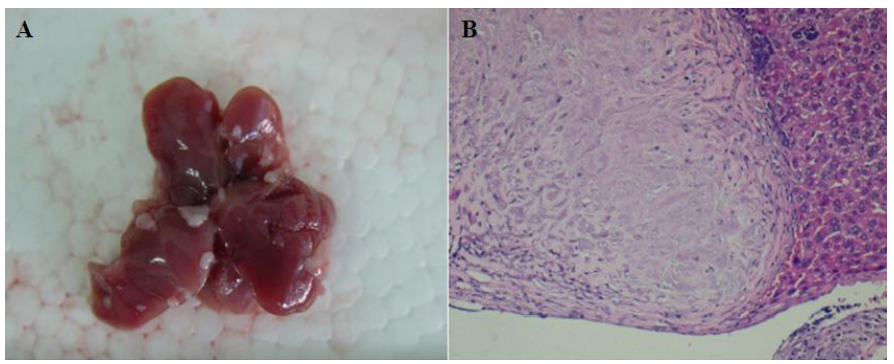

Figure 3. A. Second group = many white nodules emerged in the liver. B. Second group $=$ there were foreign body granulomas found inside of the tunication of liver.

\section{JEM-1010 transmission microscope observations}

Many phagocytes were found collected in the granuloma of the spleen and liver. In the cytoplasm, a large number of phagosomes emerged, whose single layer was full of phagocytosed substance (Figure 4A). There were two types of phagocytosed substances. One was observed under high magnification and was arranged in bundles, and the other was observed under low magnification and appeared as tiny particles. Around the phagocytes, the mitochondria were swelling and some of the mitochondrion ridges disappeared. Moreover, the double layer of some mitochondria disappeared and formed vacuole structures. Fibroblasts and several lymphocytes and phagocytes could be seen around the capillary in the granuloma tissue. There was a small amount of collagen fibers in the mesenchyme. The structure of the granuloma of the third group was similar to that of the second group except for a smaller amount of phagocytes.

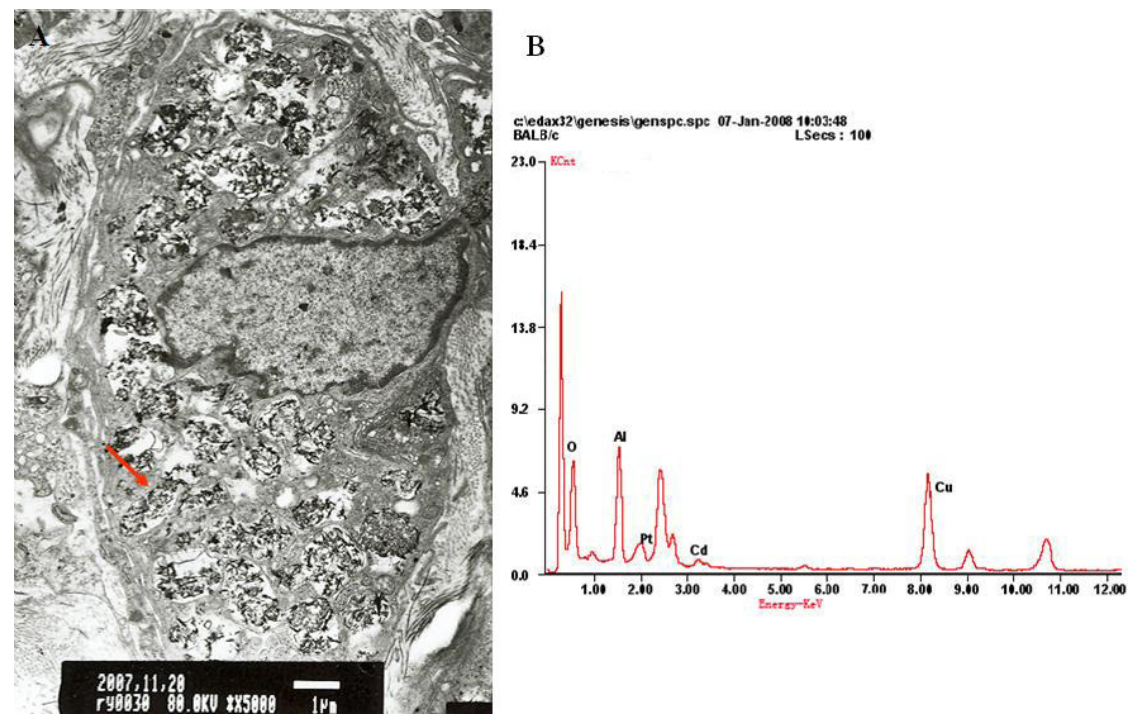

Figure 4. A. JEM-1010 observation of the second group, the arrows pointed out the needle-like structure in the cytoplasm of phagocyte $(5000 \mathrm{X})$. B. Second group $\left[\mathrm{Al}(\mathrm{OH})_{3}\right.$ gel] $=$ JEM-2100F energy spectrum analysis of spleen, the foreign body in the sample showed peak value at aluminum. 


\section{JEM-2100F energy spectrum analysis}

The electron microscope analysis showed a peak value at aluminum. This demonstrated that high magnification fine needles and oval substances and low magnification tiny particles both contained aluminum (Figure 4B).

\section{DISCUSSION}

Establishment of an animal model for AR is crucial for studying the mechanism of AR. The main principle of producing the animal model is that the induced symptoms and pathology can be easily duplicated. As the basic step for AR functional studies, a successful animal model is determined by observations of the behavior of animals and histological changes.

OVA is a protein with good antigenicity and carrier activity. Although OVA also has immunogenicity, the addition of some adjuvants are still necessary to reinforce the immunogenicity in establishing an animal model. $\mathrm{Al}(\mathrm{OH})_{3}$ is widely used in animal experiments owing to its nontoxic properties and good adsorbability. It can induce humoral immunity reactions and simulate Th2 cells to generate antibodies at high levels, and also satisfies safety requirements. At present, $\mathrm{OVA}+\mathrm{Al}(\mathrm{OH})_{3}$ is widely accepted as fundamental material for producing animal models around the word (Sehmi et al., 1997).

However, there are some problems associated with the use of $\mathrm{Al}(\mathrm{OH})_{3}$ as an adjuvant. The adjuvant should be able to effectively generate the accurate animal model while not resulting in unacceptable pathological changes. Therefore, it is better to minimize the amount of adjuvant-allergen complex used in order to induce the expected immune response and the least adverse reaction (Wiedermann-Schmidt and Maurer, 2005). Two traditional dosage forms of aluminum adjuvant are widely used by researchers. The first involves placing the aluminum suspension into the solution containing the antigen to form a protein-aluminum salt complex, and the other is putting the solution containing the antigen into $\mathrm{Al}(\mathrm{OH})_{3}$ solution or aluminum phosphate to form aluminum-containing vaccines. Recent studies found that making the aluminum adjuvant into nanometer form could induce the specific antibody in mice quickly and at a high level, and that compared with other forms of aluminum adjuvant, it could induce humoral immunity earlier (Frey et al., 1999). After consulting the relevant literature, we found the amount of OVA used in animal models generally ranges from 10 to $100 \mu \mathrm{g}$ (Yamaki et al., 2005; Tumes et al., 2007; Liu et al., 2010; Oh et al., 2011), whereas the amount of $\mathrm{Al}(\mathrm{OH})_{3}$ ranges from 1 and $5 \mathrm{mg}$. Moreover, there are several forms of $\mathrm{Al}(\mathrm{OH})_{3}$ used in these experiments including powder, gel, solution, and other dosage forms (Yamaki et al., 2005; Zhao et al., 2005; Su et al., 2006; Tumes et al., 2007; Mo et al., 2011). Our study focused on these three dosage forms (powder, gel, and solution) in the production of an AR animal model.

In the first group of mice (powder), there were no abdominal bulges and no white precipitated substance appeared. A large amount of eosinophils aggregated in the mucosa of the nasal lateral wall and inferior turbinate. Goblet cells proliferated, lymphocytes infiltrated, and the amount of plasmocytes increased. These observations demonstrated that the animal model was successfully produced. Because of the difficulty for $\mathrm{Al}(\mathrm{OH})_{3}$ powder to dissolve, we observed some powder precipitating on the bottom of the bottle, and the adjuvant-allergen complex existed in the form of suspension. Although we tried to blend the solution sufficiently, the liquid extracted in the syringe still contained relatively less $\mathrm{Al}(\mathrm{OH})_{3}$. Therefore, 
we estimated that the effective concentration was below that specifically designed [50 $\mu \mathrm{g}$ OVA $+5 \mathrm{mg} \mathrm{Al}(\mathrm{OH})_{3}$ powder]. We could then extrapolate that the concentration of the suspension was appropriate for establishing successful animal models.

Mice of the $\mathrm{Al}(\mathrm{OH})_{3}$ gel group showed toxic symptoms and foreign body granuloma was found in all ten mice. The aluminum was detected by energy spectrum analysis of the needle-like foreign body. Early evidence about the safety problem of aluminum adjuvants were discussed in a workshop focused on the use of aluminum adjuvants in vaccine acquisition that was held in Puerto Rico in May of 2000. During the meeting, the scientists discussed macrophages myofascial inflammation (MMF), which was first found in France, and then pointed out that there were many macrophages around the muscle fibers containing PAS-positive crystal structures, which were ultimately identified as aluminum. This supported that damage of the MMF was caused by the aluminum adjuvant. Thus, although aluminum adjuvants have been widely used in the production of animal models, this method still has safety problems that require attention.

In the third group of mice (solution), we used the same amount $\mathrm{Al}(\mathrm{OH})_{3}$ solvents as used in the $\mathrm{Al}(\mathrm{OH})_{3}$ gel; however, no AR symptoms were apparent, although foreign body granuloma was observed in the viscera. Compared with the $\mathrm{Al}(\mathrm{OH})_{3}$ gel, the particles of $\mathrm{Al}(\mathrm{OH})_{3}$ solvents were smaller, ranging between $1 \mathrm{~nm}$ and $100 \mathrm{~nm}$. Based on colloid chemistry, $\mathrm{Al}(\mathrm{OH})_{3}$ solvents easily form a colloidal dispersion system, in which the particles range from 1 to $100 \mathrm{~nm}$. Although there were still physical boundary surfaces because of the lower sinker rate, we could nonetheless obtain an approximate concentration compared with previous designs. Thus, we speculated that the overdose of aluminum in the third group caused the foreign body granuloma and immunosuppression instead of AR symptoms and pathological changes. Aluminum metabolism in the animals showed that the aluminum adjuvants were absorbed by citric acid in the tissue fluid and were then quickly discharged from the body.

We administrated $5 \mathrm{mg} \mathrm{Al}(\mathrm{OH})_{3}$ in three different dosage forms in 6-8 week-old female mice, and only the powder group successfully produced the allergic rhinitis animal model. We can speculate various reasons to explain these results. Because of the slight solubility of $\mathrm{Al}(\mathrm{OH})_{3}$, it will form a colloidal dispersion system. The sedimentation rate of the colloidal solid is much slower than that of the solute particles in the suspension. Although we blended the liquid well when we extracted the liquid from test tubes, we nonetheless obtained different concentrations. The gel group was set to $5 \mathrm{mg} / \mathrm{mL}$, and the concentration of the powder group was lower than that of the hydrosolvent group, and they were both lower than that of the gel group. Therefore, after the intraperitoneal injection, the extra colloidal solid could not be well absorbed and instead aggregated in the viscera, stimulating phagocytocis around the cell, and ultimately forming the foreign body granuloma. Accordingly, we speculated that excessive aluminum could cause foreign body granuloma and other adverse reactions. A previous study demonstrated that excessive aluminum could result in different kinds of inflammation caused by complement activation (Clements and Griffiths, 2002).

There are few reports available that focus on the concentration and toxicological effects of aluminum. Our animal model experiment showed that when the dosage form of aluminum adjuvant was changed, we could not simply convert the concentration because of consequent changes in the isoelectric point and physical and chemical properties, which can play important roles in influencing the results. Understanding how to use the different dosage forms of aluminum adjuvant in animal experiments could help to guarantee the safety of experimental animals and generate accurate AR animal models. 
Another interesting finding was that there was not an increase in the number of eosinophils in the nasal mucosa of mice from the gel and hydrosolvent groups, which demonstrated that extra aluminum adjuvant could induce immunosuppression and other adverse actions. To generate successful animal models, researchers need to understand the extrinsic factors (type of allergen, concentration, dosage form, and correct method of immunoadjuvant use) and animal factors (genetic background, gender, age), and so on. Ignoring any of the factors mentioned above could result in a bad outcome. In particular, scientists should pay particular attention to these factors when aluminum adjuvants are used in the establishment of animal models, which will benefit the animal experiment.

\section{CONCLUSION}

The immunoadjuvant $\mathrm{Al}(\mathrm{OH})_{3}$ was routinely administered during the establishment of AR animal models for the enhancement of the immunogenicity of antigenic substances. The dosage form of immunoadjuvants directly determined whether or not a model was successfully established, and moreover, an overdose of $\mathrm{Al}(\mathrm{OH})_{3}$ could result in immunosuppressive action, leading to foreign body granuloma and ascites of internal organs. This study is expected to provide an experimental basis for the safe use of $\mathrm{Al}(\mathrm{OH})_{3}$ in animal models and serves as a reminder of the importance and considerations in using $\mathrm{Al}(\mathrm{OH})_{3}$ in $\mathrm{AR}$ studies.

\section{Conflicts of interest}

The authors declare no conflict of interest.

\section{ACKNOWLEDGMENTS}

Research supported by the National Science Fund for Distinguished Young Scholars (\#81025007), the Beijing Natural Science Foundation (\#7131006), the Capital Health Research and Development of Special (\#2011-1017-06), the Science Foundation for High-Level Medical Talents of Beijing Health System (\#2009-02-007), the Health Ministry Special Fund (\#201202005), and the Beijing Nova Program (\#2010B022) to L. Zhang and Y. Zhang.

\section{REFERENCES}

Clements CJ and Griffiths E (2002). The global impact of vaccines containing aluminium adjuvants. Vaccine 20 Suppl. 3: S24-S33.

de Serres FJ (1988). Banbury Center DNA Adducts Workshop. Workshop on DNA Adducts, Banbury Center, Cold Spring Harbor Laboratory, Cold Spring Harbor, New York, NY (U.S.A.), Sept. 30-Oct. 2, 1986. Mutat. Res. 203: 55-68.

Frey A, Mantis N, Kozlowski PA, Quayle AJ, et al. (1999). Immunization of mice with peptomers covalently coupled to aluminum oxide nanoparticles. Vaccine 17: 3007-3019.

Liu Y, Lu X, Yu HJ, Hua XY, et al. (2010). The expression of osteopontin and its association with Clara cell $10 \mathrm{kDa}$ protein in allergic rhinitis. Clin. Exp. Allergy 40: 1632-1641.

Mo JH, Kang EK, Quan SH, Rhee CS, et al. (2011). Anti-tumor necrosis factor-alpha treatment reduces allergic responses in an allergic rhinitis mouse model. Allergy 66: 279-286.

Oh HA, Kim HM and Jeong HJ (2011). Beneficial effects of chelidonic acid on a model of allergic rhinitis. Int. Immunopharmacol. 11: 39-45.

Sehmi R, Wood LJ, Watson R, Foley R, et al. (1997). Allergen-induced increases in IL-5 receptor alpha-subunit expression 
on bone marrow-derived $\mathrm{CD} 34^{+}$cells from asthmatic subjects. A novel marker of progenitor cell commitment towards eosinophilic differentiation. J. Clin. Invest. 100: 2466-2475.

$\mathrm{Su}$ YC, Rolph MS, Cooley MA and Sewell WA (2006). Cyclophosphamide augments inflammation by reducing immunosuppression in a mouse model of allergic airway disease. J. Allergy Clin. Immunol. 117: 635-641.

Tumes DJ, Cormie J, Calvert MG, Stewart K, et al. (2007). Strain-dependent resistance to allergen-induced lung pathophysiology in mice correlates with rate of apoptosis of lung-derived eosinophils. J. Leukoc. Biol. 81: 13621373.

Wiedermann-Schmidt U and Maurer W (2005). Adjuvants and additives in vaccines-medical relevance. Wien. Klin. Wochenschr. 117: 510-519.

Yamaki K, Alam AH, Hossain MA, Taneda S, et al. (2005). Effect of rolipram, a phosphodiesterase IV inhibitor, on allergic footpad swelling using various adjuvants in mice. Scand. J. Immunol. 62: 378-384.

Zhao J, Wang S and Ma F (2005). Changes of Th1, Th2 cells and correlative cytokines in allergic rhinitis. Lin. Chuang. Er. Bi Yan Hou Ke. Za Zhi 19: 794-796. 